\title{
MAESTROS Y MAESTRAS ANTE SITUACIONES DE ACOSO Y CIBERACOSO ESCOLAR
}

\author{
Francesc Sidera \\ Universitat de Girona \\ francesc.sidera@udg.edu \\ Carles Rostan \\ Universitat de Girona \\ carles.rostan@udg.edu \\ Elisabet Serrat \\ Universitat de Girona \\ elisabet.serrat@udg.edu \\ Robinson Ortiz \\ Universitat de Girona \\ hrobinsonc@gmail.com
}

Fecha de Recepción: 21 Enero 2019

Fecha de Admisión: 30 Abril 2019

\section{RESUMEN}

El objetivo de este estudio se centra en conocer el punto de vista y comportamiento de los maestros y maestras de educación infantil y primaria en situaciones de acoso escolar. Un total de 220 docentes han participado en el estudio (182 mujeres y 38 hombres; edad media $=42.24$ años), respondiendo a un cuestionario en línea sobre algunos aspectos relacionados con el acoso escolar. Los resultados muestran que solo un $24.1 \%$ se consideran preparados para afrontar situaciones de acoso 0 ciberacoso. Además, un $45.2 \%$ afirma haber observado situaciones de acoso 0 ciberacoso en las aulas donde ha realizado docencia. En estos casos, las acciones que más frecuentemente llevan a cabo implican hablar con el agresor y la víctima, los compañeros de clase, el equipo directivo y las familias del agresor y de la víctima. Los comportamientos menos frecuentes implican castigar al agresor y hablar con los profesionales del centro, como por ejemplo, el psicólogo. Finalmente, los maestros y maestras consideran que las acciones que ayudarían más a prevenir y reducir el acoso y el ciberacoso son: concienciar sobre las consecuencias del acoso, animar a los alumnos y alumnas a informar de las situaciones de acoso, implementar programas y protocolos de actuación adecuados y formación a los docentes y a las familias. Estos resultados se discuten en el marco de otros estudios sobre la percepción y las actuaciones de los maestros y maestras en situaciones de acoso y ciberacoso escolar, así como dentro de las prácticas más efectivas para su prevención y reducción. 


\section{MAESTROS Y MAESTRAS ANTE SITUACIONES DE ACOSO Y CIBERACOSO ESCOLAR}

Palabras clave: acoso escolar; ciberacoso; maestros; maestras; prevención; reducción; actuaciones

\section{ABSTRACT}

Teachers in front of school bullying and cyberbullying. The objective of this study is to learn about the point of view and behavior of teachers of nursery school and primary education in situations of bullying. A total of 220 teachers participated in the study (182 women and 38 men; average age $=42.24$ years) responding to an online questionnaire about some aspects related to bullying. The results show that only $24.1 \%$ consider themselves prepared to face situations of bullying or cyberbullying. In addition, a $45.2 \%$ of the teachers say they have observed situations of bullying or cyberbullying in their classrooms. In these cases, the actions they carry out most often involve talking with the aggressor and the victim, with classmates, with the school management team and with the families of the aggressor and of the victim. The least frequent behaviors involves punishing the aggressor and talking with the other professionals who work in the center, such as the psychologist. Finally, teachers believe that the actions that would most help to prevent and reduce bullying and cyberbullying are: raising awareness about the consequences of bullying, encouraging students to report bullying situations, implement adequate programs and protocols, and training teachers and families. These results are discussed in the framework of other studies focused on the perception and actions of teachers in situations of bullying and cyberbullying, and on research about the most effective practices for their prevention and reduction.

Keywords: bullying; cyberbullying; teachers; prevention; reduction; actions

\section{INTRODUCCIÓN}

El bullying 0 acoso se puede describir como un abuso sistemático e intencional hacia un individuo por parte de otro individuo o un grupo (Smith, 2014), en una situación de desequilibrio de poder que puede ser real o percibida, y que causa daño o malestar físico, psicológico, social o educativo en la víctima (Gladden, Vivolo-Kantor, Hamburger, y Lumpkin, 2014). Además, este abuso no se limita a eventos espontáneos, sino que se prolonga en el tiempo y tiene además tiene un componente de planificación donde la parte agresora trata de forma tiránica al otro, hasta el punto de convertirlo en víctima habitual (Olweus, 2004). Autores como Smith y Sharp (1994) o Díaz (2006) proponen que el bullying es un tipo específico de violencia que se observa en la edad escolar y en la que se pueden identificar distintos tipos de comportamientos agresivos, como la agresividad física (por ejemplo dar puñetazos), verbal (insultar, intimidar), relacional (humillación) o digital (llamado ciberbullying; por ejemplo, colgar fotos ofensivas de alguien en Internet). Todos estos tipos de agresividad pueden ocurrir de forma directa, es decir, en presencia de la víctima, o de forma indirecta, en su ausencia (ej., difundir rumores). Así pues, en las formas indirectas el agresor puede permanecer sin identificar por la víctima. Psicológicamente, las formas indirectas pueden representar una mayor afectación, ya que la víctima puede tener sentimientos de autoinculpación, al no atribuir una causa externa a su sufrimiento. Las formas directas suelen ser más propias de los chicos, mientras que las indirectas son más usuales en las chicas (Smith, 2014).

La investigación ha demostrado que una falta de conciencia y una intervención inefectiva en situaciones de bullying pueden llevar a consecuencias a largo plazo tales como bajo rendimiento académico en agresores y víctimas, depresión, sentimiento de inutilidad, miedo y rechazo al contexto escolar en las víctimas (Olweus, 2004), y comportamiento antisocial en los agresores. Así pues, dado que el acoso escolar puede tener consecuencias psicológicas graves tanto en los agresores como en las víctimas (UNESC0, 2017), que pueden mantenerse a lo largo de la vida (Stuart y Jose, 2014), se hace necesario seguir investigando en formas de prevención e intervención eficaces. 
Uno de los actores claves en el acoso escolar son los maestros y maestras, ya que tienen un papel fundamental en la identificación, prevención e intervención. Sin embargo, los maestros pueden tener dificultades para identificar comportamientos de bullying, porque a menudo implican comportamientos encubiertos, porque los efectos en las víctimas no son observables de forma inmediata, y porque se requiere de una comprensión adecuada de cómo los comportamientos en situaciones de acoso pueden variar según la edad del alumnado y sus características individuales (DeOrnellas y Spurgin, 2017). Por otra parte, sabemos que los maestros se pueden beneficiar de los programas de prevención y protocolos de intervención contra el bullying, ya que les puede dotar de herramientas para su detección y para la mejora del clima escolar (Bowllan, 2011). Así pues, es importante saber hasta qué punto los maestros y maestras conocen la existencia de estos protocolos y programas.

\section{OBJETIVOS}

Dado el importante papel que juegan los maestros y las maestras en situaciones de acoso y ciberacoso, en el presente estudio pretendemos examinar cuál es su conocimiento y percepción sobre este fenómeno, cómo actúan cuando se encuentran este tipo de situaciones en su aula, y cuáles son sus propuestas para la prevención e intervención.

\section{MÉTODO}

\section{Participantes}

Este trabajo forma parte de un estudio de prevalencia sobre acoso y ciberacoso, donde llevó a cabo una recogida de datos en una muestra representativa de alumnado de $3^{\circ}$ a $6^{0}$ de primaria proveniente de 41 escuelas de Cataluña. Un total de 220 maestros (182 mujeres y 38 hombres) de esas mismas escuelas que respondieron a un cuestionario sobre acoso escolar conforman la muestra del presente trabajo. La media de edad de los participantes fue de 42.24 años $(D T=9.65)$ y los años de experiencia como maestros 17.51 años $(D T=17.51)$.

\section{Instrumentos}

Se diseñó un cuestionario de acoso y ciberacoso escolar para maestros, en lengua catalana y castellana (ver apéndice) compuesto por 12 preguntas, de las cuáles hemos analizado aquí sólo aquellas relacionadas con: a) el conocimiento de los maestros sobre la existencia en su escuela de protocolos de prevención y programas de intervención; b) la formación recibida sobre acoso escolar en su escuela; c) su percepción sobre si están preparados para afrontar situaciones de acoso escolar; d) qué apoyos y recursos consideran necesarios para afrontar el acoso; e) la observación de acoso en sus aulas, y qué actuaciones han llevado a cabo; f) su punto de vista sobre qué acciones ayudarían a prevenir y reducir el acoso escolar.

\section{Procedimiento}

El cuestionario electrónico fue enviado vía correo electrónico a los equipos directivos de los centros educativos que participaron en el estudio, que se encargaron de distribuirlo al equipo docente de Educación Primaria del centro. Antes de acceder al cuestionario en línea, los maestros debían firmar telemáticamente un documento de consentimiento informado.

\section{RESULTADOS}

\section{Programas de prevención y protocolos de actuación}

Tal como se puede observar en la Figura 1, el porcentaje de maestros que afirman que en su 
escuela hay programas de prevención de bullying o ciberbullying es inferior al $30 \%$, siendo un poco más alto en el caso de los protocolos de actuación. Así pues, la mayoría de maestros afirman que en su escuela no hay programas de prevención o protocolos de actuación, o bien desconocen su existencia.

Figura 1. Conocimiento sobre la existencia de programas de bullying y ciberbullying (porcentaje de respuestas).

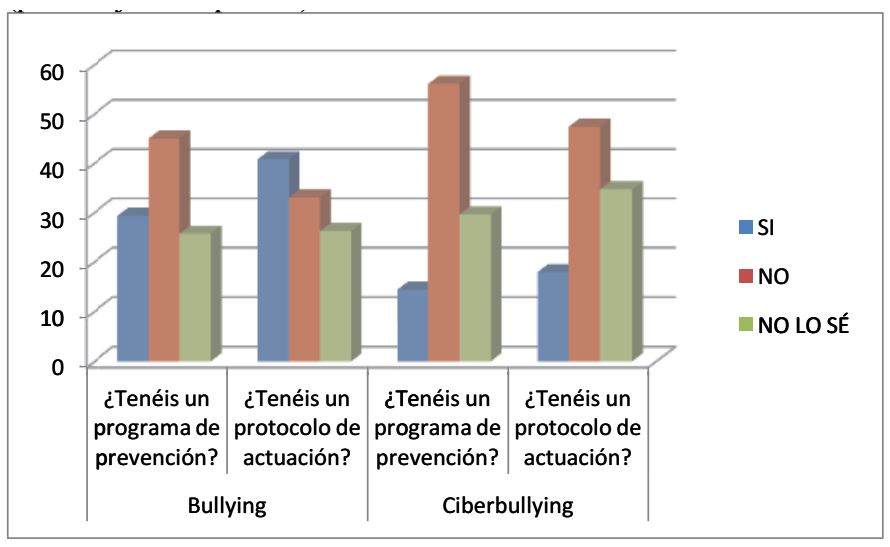

En relación con la pregunta realizada a los maestros sobre si habían recibido formación sobre estos programas o protocolos, del total de respuestas ( $N=163)$ un $35 \%$ afirmaron que habían recibido formación, pero el $65 \%$ no.

\section{Percepción de preparación para situaciones de acoso}

En cuanto a si los maestros se consideran preparados para afrontar situaciones de bullying 0 ciberbullying, la mayoría respondieron $(\mathrm{N}=162)$ que no están seguros de ello $(61.7 \%)$, y otros manifestaron que no están preparados (14.2\%). Así, solamente un $24.1 \%$ afirman estar preparados para afrontar situaciones de bullying 0 ciberbullying.

\section{Apoyos y recursos para afrontar situaciones de bullying en la escuela}

Cuando se preguntó a los participantes sobre si consideran que tienen los recursos y apoyos suficientes para afrontar este tipo de situaciones, los maestros respondieron mayoritariamente que no están seguros (45\%) o que no tienen recursos o apoyos suficientes $(37.17 \%)$, y solo un $17.7 \%$ de los maestros creen que sí.

En cuanto a la pregunta abierta sobre qué apoyos o recursos los maestros creen que se necesitan en su centro per mejorar la convivencia y prevenir las situaciones de bullying y ciberbullying, las respuestas se agruparon en las categorías siguientes:

Apoyos o recursos relacionados con el Departamento de Educación.

Dotar de asesoramiento al equipo docente (por ejemplo, aumentar las horas de los psicólogos en la escuela) y permitir que los maestros tengan contacto directo con los psicólogos.

Realizar formación a los maestros. Por ejemplo, a través de casos de éxito.

Dotar a los docentes de horas parar tratar al alumnado afectado.

Bajar las ratios en las aulas.

Que un profesional visite los centros cuando haya casos de bullying. 
Fomentar que la escuela trabaje conjuntamente con los servicios sociales y los servicios de salud mental.

Actuaciones en el barrio (y en las entradas de las escuelas) para mejorar la seguridad.

Apoyos o recursos relacionados con el centro educativo y el equipo docente.

Que los centros desarrollen y actualicen protocolos, programas, pautas o prácticas para detectar y afrontar situaciones de bullying o ciberbullying, con la colaboración de los claustros.

Que el profesorado disponga de material para trabajar con el alumnado (dinámicas, textos, situaciones, vídeos, actividades...).

Coordinación entre los tutores del centro y la comisión de convivencia.

Crear equipos de mediación en la escuela formados por maestros y alumnos.

Aumentar el control fuera de horas de clase (ej., comedor).

Tener medidas de expulsión firmes.

Limitar el uso de los chats en casa y en la escuela.

Formar y asesorar a las familias (por ejemplo, sobre el control parental en las nuevas tecnologías)

Implicar a las familias en las actuaciones de la escuela.

Que la escuela se implique en los problemas que ocurren al alumnado fuera de la escuela.

Apoyos o recursos relacionados con el alumnado

Potenciar las tutorías con el alumnado, a nivel grupal e individual, desde la etapa de Educación Infantil.

Fomentar la convivencia entre el alumnado: que se dé importancia a las relaciones y a aprender a convivir juntos, trabajar la educación emocional, fomentar la tolerancia, la cooperación y el respeto por la diversidad.

Realizar charlas participativas y crear espacios donde el alumnado se pueda expresar libremente.

Crear una comisión donde también participe el alumnado.

Realización de sociogramas con el alumnado.

Soporte y acompañamiento personal a las víctimas, y trabajo con los agresores.

Al preguntar sobre si se realizan formaciones periódicas en el centro orientadas al alumnado para prevenir o reducir el bullying 0 el ciberbullying, la mayoría de maestros respondieron que no (77.2\%), mientras que el resto respondieron que sí (22.8\%).

\section{Actuaciones ante el bullying y ciberbullying}

Al preguntar a los maestros si han observado alguna vez situaciones de bullying en las aulas donde realizan docencia, casi la mitad de los maestros (45.2\%; $N=99$ ) han respondido que sí, mientras que el $54.8 \%$ que no. A los maestros que respondieron afirmativamente les preguntamos cómo habían actuado en esa situación. (Ver Tabla 1). 
Tabla 1. Actuaciones ante situaciones de bullying y cibebullyinga.

\begin{tabular}{lc} 
Acción & $\mathbf{\%}$ \\
\hline Hablar con el agresor & 87,9 \\
Hablar con la víctima & 84,8 \\
Hablar con los compañeros de clase & 83,8 \\
Hablar con el equipo directivo & 82,8 \\
Hablar con los padres del agresor & 75,8 \\
Hablar con los padres de la víctima & 68,7 \\
Hablar a la vez con agresor y víctima & 59,6 \\
Hablar con otros profesionales del centro & 46,5 \\
Castigar al agresor & 37,4
\end{tabular}

${ }^{a}$ Porcentaje de maestros que han realizado cada una de las acciones indicadas.

Aparte de las opciones de respuesta ya dadas, un total de 12 maestros indicaron otras acciones que han realizado en estas situaciones. Son las siguientes:

Coordinarse con los responsables del comedor para supervisar las horas donde el maestro no está presente.

Formarse.

Realizar actividades o dinámicas de grupo en el aula.

Proponer una mediación.

Crear espacios para que el alumnado pueda hablar.

Trabajo de autoestima y de perdón.

Hablar con el claustro.

Derivar los casos con trastorno al centro de salud mental.

Solicitar a la policía la realización de una charla en clase.

Finalmente, se solicitó a los maestros que valorasen algunas acciones en función de si estas ayudarían a prevenir y/o reducir el bullying y ciberbullying en sus centros (ver Tabla 2). Así pues, los maestros debían valorar cada acción en una escala que iba desde "No ayudaría" (1 punto) hasta "Ayudaría mucho" (5 puntos). Aquellas acciones que se valoraron como menos positivas fueron aumentar el castigo a los agresores, limitar el acceso de los estudiantes a los medios electrónicos, aumentar la supervisión de algunos lugares como el patio de la escuela, o mejorar las relaciones entre maestros. 
Tabla 2. Acciones que ayudarían a mejorar la situación de convivencia del centro educativo, así como a prevenir y/o reducir el bullying y ciberbullyinga.

\begin{tabular}{lc}
\hline Acción & $\mathbf{\%}$ \\
\hline Concienciar sobre las consecuencias del acoso & 4,56 \\
Alentar al alumnado para que informen de situaciones de acoso & 4,42 \\
Implementar programas de prevención adecuados & 4,33 \\
Implementar protocolos de actuación adecuados & 4,29 \\
Realizar seminarios de formación específicos con los maestros & 4,24 \\
Educar a los padres para que apoyen al estudiante & 4,22 \\
Aumentar la implicación de los padres en la escuela & 4,16 \\
Mejorar la relación entre los maestros y el alumnado & 4,01 \\
Hacer asambleas escolares anti-bullying & 4,08 \\
Realizar clases anti-bullying con el alumnado & 4,02 \\
Establecer un grupo de trabajo sobre acoso escolar & 3,82 \\
Mejorar las relaciones entre los maestros & 3,61 \\
Aumentar la supervisión de algunos lugares & 3,51 \\
Limitar el acceso a los medios electrónicos & 3,26 \\
Aumentar los castigos a los agresores & 2,67 \\
\hline${ }^{2}$ Media de las puntuacion obteidas.
\end{tabular}

${ }^{\mathrm{a}}$ Media de las puntuaciones obtenidas.

Al preguntar sobre otras propuestas para prevenir y reducir el bullying y ciberbullying, las respuestas recibidas por 38 participantes se agruparon en las siguientes categorías:

Trabajo con la organización de los centros educativos

Bajar las ratios de las aulas.

Aumentar la presencia de maestros en el patio.

Aumentar el personal con formación psicopedagógica en los centros.

Evitar que el alumnado esté solo fuera de la escuela (ex: impulsar actividades desde los ayuntamientos).

Iniciar el trabajo de prevención desde el Ciclo Inicial.

Trabajo con el alumnado

Cohesionar al alumnado. 
Realizar tutorías individuales y grupales.

Implicar al alumnado en la lucha contra el bullying (por ejemplo, concienciar sobre la importancia de los observadores).

Crear canales de comunicación para que los observadores puedan informar de situaciones de bullying sin ser juzgados.

Organizar campañas escolares.

Tratar el concepto de bullying y ciberbullying con el alumnado.

Trabajar la educación emocional y la empatía con la víctima.

Romper los esquemas competitivos y valorar el trabajo en equipo.

Dotar de asistencia psicológica a los agresores.

Educar en el uso de los medios electrónicos.

Traer testimonios de situaciones de bullying al alumnado.

Trabajo con les familias

Implicar a las familias en el trabajo que se realiza en la escuela.

Realizar charlas con las familias.

Concienciar al alumnado y a las familias del daño que pueden causar a las víctimas, así como de los motivos de sus agresiones.

Concienciar a las familias que sus hijos pueden ser agresores.

\section{DISCUSIÓN}

De los datos recogidos en los cuestionarios se desprende que es necesario mejorar mucho el conocimiento que tienen los maestros sobre los programas de prevención y protocolos de intervención de acoso escolar, y especialmente de ciberacoso. Estos datos chocan con el hecho de que, desde junio de 2018, el Departament d'Ensenyament de la Generalitat de Catalunya tiene disponible en su página web el documento "Protocolo de prevención, detección e intervención delante del acoso y ciberacoso entre iguales". Por tanto, un primer paso a realizar es dar a conocer este protocolo al conjunto de la comunidad docente. Ante la incidencia que alcanza el fenómeno, quizás convendría realizar presentaciones en las escuelas y animar a las direcciones a sensibilizar a los maestros de la importancia que tiene esta problemática tanto para el bienestar de los alumnos como para el buen clima de la escuela para el aprendizaje. También es necesario que los claustros de las escuelas desarrollen este protocolo, lo actualicen, y lo adapten a la realidad de cada centro.

Uno de los aspectos que han destacado los maestros de este estudio como necesario para luchar contra el bullying ha sido el de la formación. Es un hecho común que los maestros se den cuenta de la necesidad de formarse sobre el bullying (DeOrnellas y Spurgin, 2017). En consecuencia, es crucial plantearse qué contenidos son necesarios trabajar ya desde la formación inicial de maestros.

Hay que tener en cuenta que algunos estudios indican que la percepción del bullying es muy variable de unos maestros a otros, y que esta percepción tiene una influencia en cómo los maestros actúan. Por ejemplo, algunos maestros creen que los comportamientos implicados en el bullying son típicos en determinadas etapas educativas, que las situaciones de acoso pueden hacer fuertes a las víctimas 0 ayudarlas a madurar, o que los niños podrán resolver la situación por sí mismos. Además, algunos maestros tienen dificultades para diferenciar el acoso de las bromas no mal intencionadas, y en ocasiones culpabilizan a las víctimas para permitir que estas situaciones continúen. Por otra parte, algunos maestros atribuyen el hecho de ser agresor a determinados factores familiares, lo que puede llevar a situar la responsabilidad de los agresores fuera del escuela (ver DeOrnellas y Spurgin, 2017). Así pues, la formación de maestros debe ir dirigida no sólo a conocer cómo actuar en situaciones de bullying, sino también a trabajar sus percepciones sobre este fenó- 
meno, y su capacidad de identificar y diferenciar comportamientos de bullying. En efecto, los maestros parecen tener más dificultades para identificar como bullying comportamientos de agresividad indirecta o relacional (Mishna, Scarcello, Pepler, y Weiner, 2005) y suelen intervenir más cuando los comportamientos son de agresividad descubierta (Waasdorp, Pas, O'Brennan, y Bradshaw, 2011). El porcentaje de maestros de nuestro estudio que afirman no haber observado nunca una situación de bullying podría apoyar esta idea de la necesidad de formar a los maestros en la detección.

Los docentes también necesitan sentirse competentes para afrontar el acoso y el ciberacoso. En este sentido, solo un $24.1 \%$ de los maestros de nuestro estudio manifiestan sentirse preparados. Este dato es muy relevante, ya que uno de los factores que influyen en la probabilidad de que un maestro intervenga en una situación de agresividad escolar es precisamente la confianza que éste tiene para solucionar este tipo de problemas (Alvarez, 2007; Acquadro Maran, Tirassa y Begotti, 2017). Así pues, nuestro estudio muestra que es necesaria una formación específica que dé seguridad a los maestros sobre cómo intervenir.

Por otra parte, pocos maestros perciben tener el apoyo necesario para afrontar el acoso y el ciberacoso. Por tanto, se necesita una coordinación estrecha entre los profesionales involucrados en el bullying y el ciberbullying para crear y llevar a cabo programas de prevención e intervención eficaces y eficientes. Además, las instituciones deben plantearse la necesidad de aumentar la presencia de determinados profesionales en las escuelas para formar a los maestros, apoyar a las tutorías, acompañar a víctimas y agresores, implicar a las familias, etc.

Por último, la riqueza de respuestas a la pregunta abierta sobre qué recursos se necesitan para mejorar la convivencia sugiere la necesidad de empoderar a las maestras como agentes activos de los programas de prevención e intervención, ya que son un factor clave para su éxito (Kyriakides y Creemers, 2012). Algunas de las ideas aportadas en este sentido incluyen impulsar actividades fuera de la escuela desde los ayuntamientos, implicar a tota la comunidad educativa en la lucha contra el acoso escolar, educar en el uso de los medios electrónicos, o iniciar el trabajo de prevención desde las primeras etapas educativas.

\section{Agradecimientos}

Este estudio se ha llevado a cabo gracias a la financiación de la Fundación Fútbol Club Barcelona (Fundación Barça). Agradecemos la colaboración de todos los centros educativos participantes en el mismo, así como a todos los maestros que respondieron al cuestionario de forma desinteresada.

\section{REFERENCIAS BIBLIOGRÁFICAS}

Acquadro Maran, D., Tirassa, M. y Begotti, T. (2017). Teachers' Intervention in School Bullying: A Qualitative Analysis on Italian Teachers. Frontiers in Educucation, 2:36.

Alvarez, H. K. (2007). The impact of teacher preparation on responses to student aggression in the classroom. Teaching and Teacher Education, 23(7), 1113-1126.

Bowllan, N. M. (2011). Implementation and evaluation of a comprehensive, school-wide bullying prevention program in an urban/suburban middle school. Journal of School Health, 81(4), 167-173.

DeOrnellas, K., y Spurgin, A. (2017). Teachers' perspectives on bullying. In L. H. Rosen, K. DeOrnellas, y S. R. Scott (Eds.), Bullying in schools: Perspectives from school staff, students, and parents (pp. 49-68). New York, NY: Palgrave Macmillan. http://dx.doi.org/10.1057/978-1137-59298-9_3

Departament d'Ensenyament de la Generalitat de Catalunya (2018). Protocol de prevenció, detecció i intervenció davant l'assetjament $i$ el ciberassetjament entre iguals. 
Díaz, M. (2006). Del acoso escolar a la cooperación en las aulas. Madrid: Persson Educación, S.A. Gladden R. M., Vivolo-Kantor A. M., Hamburger M. E., y Lumpkin C. D. (2014). Bullying surveillance among youths: Uniform definitions for public health and recommended data elements, version 1.0. Atlanta, GA: National Center for Injury Prevention and Control, Centers for Disease Control and Prevention and U.S. Department of Education.

Kyriakides, L., y Creemers, B. P. M. (2012). Characteristics of effective schools in facing and reducing bullying. School Psychology International, 34, 348-368.

Mishna, F., Scarcello, I., Pepler, D., y Wiener, J. (2005). Teachers' Understanding of Bullying. Canadian Journal of Education, v28 n4 p718-738.

Olweus, D. (2004). Conductas de acoso y amenaza entre escolares. Ediciones Morata.

Smith, P. K., y Sharp, S. (Eds.). (1994). School bullying: Insights and perspectives. London: Routledge.

Smith, P. K. (2014). Understanding school bullying. London: Sage Publications.

Stuart, J., y Jose, P. E. (2014). Is bullying bad for your health? The consequences of bullying perpetration and victimization in childhood on health behaviors in adulthood. Journal of aggression, conflict and peace research, 6(3), 185-195.

UNESCO (2017). School violence and bullying: Global status report. France: Paris.

Waasdorp, T. W., Pas, E. T., O'Brennan, L. M., y Bradshaw, C. P. (2011). A multilevel perspective on the climate of bullying: Discrepancies among students, school staff, and parents. Journal of School Violence, 10, 115-132. 


\section{APÉNDICE.}

\section{Cuestionario de bullying i ciberbullying para maestros}

Estimado/a,

Le pedimos su colaboración como maestro/a del centro mediante un cuestionario electrónico, que tiene como objetivo estudiar el punto de vista de los maestros sobre el fenómeno del acoso (bullying) y del ciberacoso (ciberbullying).

Los datos obtenidos serán tratados de forma totalmente anónima y confidencial.

¡Muchísimas gracias por su colaboración!

Antes de empezar, consideramos conveniente definir el bullying y el ciberbullying:

Bullying:

Una forma de maltrato físico, verbal o de exclusión en que el agresor / a (o agresores / as) siempre tiene más poder que la víctima. Incluye muchos tipos de comportamientos, como pegar a alguien, insultarle, criticarlo, excluirlo. Para ser considerados bullying, estos comportamientos se deben repetir durante un tiempo.

\section{Ciberbullying:}

Es un comportamiento de bullying llevado a cabo a través de medios electrónicos (ej. Móvil o Internet).

Código del centro:

Edad:

Género

Años de experiencia laboral como maestro / a:

1. ¿Tenéis protocolos de actuación o programas de prevención de bullying o ciberbullying en tu escuela?

\begin{tabular}{|l|l|l|}
\hline & SÍ & NO \\
\hline Un programa de prevención de bullying & & \\
\hline Un programa de prevención de ciberbullying & & \\
\hline Un protocolo de actuación en situaciones de bullying & & \\
\hline $\begin{array}{l}\text { Un protocolo de actuación en situaciones de } \\
\text { ciberbullying }\end{array}$ & & \\
\hline
\end{tabular}

2. ¿Has recibido formación sobre estos programas o protocolos?

O Sí

O No

\section{Responde si estás de acuerdo o no con las siguientes afirmaciones}




\begin{tabular}{|c|c|c|c|c|c|}
\hline & $\mathbf{1}$ & $\mathbf{2}$ & $\mathbf{3}$ & $\mathbf{4}$ & $\mathbf{5}$ \\
\hline $\begin{array}{c}\text { Totalmente } \\
\text { en } \\
\text { desacuerdo }\end{array}$ & $\begin{array}{c}\text { En } \\
\text { desacuerdo }\end{array}$ & $\begin{array}{c}\text { Ni de } \\
\text { acuerdo ni } \\
\text { en } \\
\text { desacuerdo }\end{array}$ & $\begin{array}{c}\text { De } \\
\text { acuerdo }\end{array}$ & $\begin{array}{c}\text { Totalmente } \\
\text { de acuerdo }\end{array}$ \\
\hline $\begin{array}{c}\text { Los programas de } \\
\text { prevención que } \\
\text { tenemos son } \\
\text { adecuados }\end{array}$ & & & & & \\
\hline $\begin{array}{c}\text { Los protocolos de } \\
\text { actuación que } \\
\text { tenemos son } \\
\text { adecuados }\end{array}$ & & & & & \\
\hline
\end{tabular}

4. ¿Te consideras preparado o preparada para afrontar situaciones de bullying o ciberbullying?
O Sí
O No
O No estoy seguro/a

5. ¿Consideras que tienes el apoyo y recursos necesarios para afrontar situaciones de bullying y ciberbullying?
O Sí
O No
O No estoy seguro/a

6. ¿Qué apoyos o recursos crees que se necesitan en tu centro para poder mejorar la convivencia y prevenir las situaciones de bullying y ciberbullying?

7. ¿Cuáles crees que son los motivos principales por los que los alumnos/as realizan bullying o ciberbullying?

8. ¿Se realizan formaciones en tu centro de manera periódica orientadas al alumnado para prevenir o reducir el bullying o ciberbullying?

O Sí

O No

9. ¿Has observado bullying o ciberbullying alguna vez con alumnos de las aulas donde haces docencia?

O Sí

O No

10. En caso de haber observado bullying o ciberbullying, ¿Cuáles de las siguientes acciones has llevado a cabo? (Puedes marcar más de una respuesta)

O Castigar al agresor/a.

O Hablar con el agresor/a. 
O Hablar con la víctima.

O Hablar con el agresor y la víctima a la vez.

O Hablar de la situación con los padres del agresor/a.

O Hablar de la situación con los padres de la víctima.

O Hablar del incidente con el equipo directivo.

O Hablar con otros profesionales del centro (ej. psicólogo)

O Otras:

\section{1. ¿Cómo te han afectado las situaciones de acoso y ciberacoso a ti y a tu clase?}

12. ¿Cuáles de las siguientes acciones crees que ayudarían a mejorar la situación de convivencia en tu centro, así como a prevenir y / o reducir el bullying y ciberbullying?

\begin{tabular}{|l|c|c|c|c|c|}
\hline & $\mathbf{1}$ & $\mathbf{2}$ & $\mathbf{3}$ & $\mathbf{4}$ & $\mathbf{5}$ \\
\hline $\begin{array}{l}\text { Numentar la supervisión } \\
\text { de algunos lugares (ej. } \\
\text { Patio) }\end{array}$ & $\begin{array}{c}\text { Ayudaría } \\
\text { poco }\end{array}$ & $\begin{array}{c}\text { Ayudaría } \\
\text { un poco }\end{array}$ & $\begin{array}{c}\text { Ayudaría } \\
\text { bastante }\end{array}$ & $\begin{array}{c}\text { Ayudaría } \\
\text { mucho }\end{array}$ \\
\hline $\begin{array}{l}\text { Implementar programas de } \\
\text { prevención adecuados }\end{array}$ & & & & & \\
\hline $\begin{array}{l}\text { Implementar protocolos de } \\
\text { actuación adecuados }\end{array}$ & & & & & \\
\hline $\begin{array}{l}\text { Hacer asambleas escolares } \\
\text { anti-bullying que } \\
\text { impliquen a toda la } \\
\text { comunidad educativa }\end{array}$ & & & & & \\
\hline $\begin{array}{l}\text { Alentar a los estudiantes } \\
\text { para que informen de } \\
\text { situaciones de bullying y } \\
\text { ciberbullying }\end{array}$ & & & & & \\
\hline $\begin{array}{l}\text { Establecer un grupo de } \\
\text { trabajo de acoso escolar en } \\
\text { el que se desarrollen } \\
\text { políticas anti-bullying }\end{array}$ & & & & & \\
\hline $\begin{array}{l}\text { Hacer clases anti-bullying } \\
\text { con el alumnado }\end{array}$ & & & & & \\
\hline $\begin{array}{l}\text { Hacer seminarios de } \\
\text { formación específicos con } \\
\text { los maestros }\end{array}$ & & & & & \\
\hline
\end{tabular}




\begin{tabular}{|l|l|l|l|l|l|}
\hline $\begin{array}{l}\text { Aumentar los castigos a } \\
\text { los agresores }\end{array}$ & & & & & \\
\hline $\begin{array}{l}\text { Crear conciencia sobre las } \\
\text { consecuencias del bullying } \\
\text { y ciberbullying }\end{array}$ & & & & & \\
\hline $\begin{array}{l}\text { Aumentar la implicación } \\
\text { de los padres en la escuela }\end{array}$ & & & & & \\
\hline $\begin{array}{l}\text { Mejorar la relación entre } \\
\text { los maestros y el } \\
\text { alumnado }\end{array}$ & & & & & \\
\hline $\begin{array}{l}\text { Mejorar las relaciones } \\
\text { entre los maestros }\end{array}$ & & & & & \\
\hline $\begin{array}{l}\text { Educar a los padres para } \\
\text { que apoyen al estudiante }\end{array}$ & & & & & \\
\hline $\begin{array}{l}\text { Limitar el acceso de los } \\
\text { estudiantes a los medios } \\
\text { electrónicos }\end{array}$ & & & & & \\
\hline \begin{tabular}{l} 
Otras: \\
\hline
\end{tabular}
\end{tabular}

Aquí puedes especificar otras ideas que tengas sobre cómo se puede prevenir y / o reducir el bullying y ciberbullying:

¡Muchas gracias por su colaboración! 